\title{
A New Lifetime Model: The Gamma Extended Fréchet Distribution
}

\author{
Ronaldo V. da Silva*, Thiago A. N. de Andrade, Diego B. M. Maciel, \\ Renilma P. S. Campos, and Gauss M. Cordeiro \\ Federal University of Pernambuco, Brazil
}

Received 14 January 2012
Accepted 11 September 2012

\begin{abstract}
For the first time, a four-parameter lifetime model, called the gamma extended Fréchet distribution, is defined and studied. We obtain some of its mathematical properties. Explicit expressions for the ordinary and incomplete moments, quantile function, mean deviations, Rényi entropy and reliability are provided. The order statistics and their moments are derived. The method of maximum likelihood is used for estimating the model parameters. We determine the observed information matrix. An application to a real data set shows that the new distribution can provide a better fit than other classical lifetime models. We hope that this generalization may attract wider applications in reliability, biology and survival analysis.
\end{abstract}

Keywords: Fréchet distribution; gamma extended Fréchet distribution; maximum likelihood; observed information matrix; Weibull distribution.

\section{Introduction}

In probability theory and statistics, the extreme value (EV) distribution is a family of continuous distributions developed within extreme value theory to combine the Gumbel, Fréchet and Weibull distributions also known as type I, II and III extreme value distributions, respectively. The EV distributions appear as limiting distributions for minimum or maximum of a sample of independent, identically distributed random variables, according to the increase of the samples size. Extreme value is the theory of modeling and measuring events occurring with very small probability. These distributions and their generalized forms are largely used in finance, economics, material sciences, telecommunications, and many others. Fréchet (or type II EV) distribution has been useful for modeling of market-returns which are often heavy-tailed in applications to finance (Alves, 2010). The cumulative distribution function (cdf) of the standard Fréchet distribution (for $x>0$ ) is

$$
G(x)=\exp \left[-\left(\frac{\sigma}{x}\right)^{\lambda}\right]
$$

where $\sigma>0$ and $\lambda>0$.

\footnotetext{
* Address for correspondence: Universidade Federal de Pernambuco, UFPE, 50740-540, Recife - Pernambuco - Brazil. e-mail: ronaldovenanciorvs@gmail.com
} 
The exponentiated exponential (EE) distribution was pioneered by Gupta and Kundu (1998) as a generalization of the standard exponential distribution (Nadarajah and Kotz, 2003). The EE cdf (for $x>0, \lambda>0$ and $\alpha>0$ ) is defined by

$$
G(x)=[1-\exp (-\lambda x)]^{\alpha},
$$

which is simply the $\alpha$ th power of the standard exponential cdf. Gupta and Kundu $(1999,2001)$ studied the mathematical properties of the EE distribution.

Later, Nadarajah and Kotz (2003) proposed the extended Fréchet (EF) distribution to generalize the standard Fréchet distribution (1) and studied some of its structural properties. The EF cdf is given by

$$
G(x ; \alpha, \lambda, \sigma)=1-\left\{1-\exp \left[-\left(\frac{\sigma}{x}\right)^{\lambda}\right]\right\}^{\alpha}
$$

for $\alpha>0$. They called (1.3) the exponentiated Fréchet model, but we adopt the EF terminology since (1.3) is not in the typical exponentiated form. The probability density function (pdf) corresponding to $(1.3)$ is

$$
g(x ; \alpha, \lambda, \sigma)=\alpha \lambda \sigma^{\lambda} x^{-(\lambda+1)} \exp \left[-\left(\frac{\sigma}{x}\right)^{\lambda}\right]\left\{1-\exp \left[-\left(\frac{\sigma}{x}\right)^{\lambda}\right]\right\}^{\alpha-1} .
$$

Zografos and Balakrishnan (2009) and Ristic and Balakrishnan (2011) proposed a family of univariate distributions generated by gamma random variables. For any baseline $\operatorname{cdf} G(x)$, and $x \in \mathbb{R}$, they defined the gamma- $G$ distribution with an extra shape parameter $a>0$ and pdf $f(x)$ and cdf $F(x)$ given by

$$
f(x)=\frac{1}{\Gamma(a)}\{-\log [1-G(x)]\}^{a-1} g(x)
$$

and

$$
F(x)=\frac{\gamma(a,-\log [1-G(x)])}{\Gamma(a)}=\frac{1}{\Gamma(a)} \int_{0}^{-\log [1-G(x)]} t^{a-1} \mathrm{e}^{-t} d t,
$$

respectively, where $g(x)=d G(x) / d(x), \Gamma(a)=\int_{0}^{\infty} t^{a-1} \mathrm{e}^{-t} d t$ denotes the gamma function and $\gamma(a, z)=\int_{0}^{z} t^{a-1} \mathrm{e}^{-t} d t$ denotes the incomplete gamma function.

Providing a new class of distributions is always precious for statisticians. Thus, the aim of this paper is to study the called gamma extended Fréchet (GEF) distribution by inserting (1.3) in equation (1.6). The fact that the GEF distribution generalizes existing commonly used distributions is an important aspect of the model. Further, we demonstrate that the proposed model provides a significant improvement compared to some existing lifetime models and it is also a competitive model to the widely used exponentiated Weibell (Mudholkar and Srivastava, 1993) distribution. In addition, we investigate some mathematical properties of the new model, discuss maximum likelihood estimation of its parameters and derive the observed information matrix.

The paper is outlined as follows. In Section 2, we define the GEF distribution and plot the density and hazard rate functions. Explicit expressions for the density and cumulative functions, ordinary and incomplete moments, quantile function, mean deviations, Rényi entropy and reliability 
are derived in Section 3. The order statistics are investigated in Section 4. We discuss maximum likelihood estimation of the model parameters in Section 5. An application in Section 6 shows the potentiality of the new distribution for lifetime data modeling. Finally, concluding remarks are given in Section 7.

\section{The GEF distribution}

The GEF distribution is defined by the cdf (for $x>0$ )

$$
F(x)=\frac{1}{\Gamma(a)} \gamma\left(a,-\alpha \log \left[1-\exp \left(-\left(\frac{\sigma}{x}\right)^{\lambda}\right)\right]\right) .
$$

The pdf and hazard rate function (hrf) corresponding to (2.1) are given by

$$
\begin{aligned}
f(x)= & \frac{\alpha \lambda \sigma^{\lambda}}{\Gamma(a)} x^{-(\lambda+1)} \exp \left[-\left(\frac{\sigma}{x}\right)^{\lambda}\right]\left\{1-\exp \left[-\left(\frac{\sigma}{x}\right)^{\lambda}\right]\right\}^{\alpha-1} \\
& \times\left\{-\log \left[1-\exp \left(-\left(\frac{\sigma}{x}\right)^{\lambda}\right)\right]^{\alpha}\right\}^{a-1}
\end{aligned}
$$

and

$$
\tau(x)=\frac{\alpha \lambda \sigma^{\lambda} x^{-(\lambda+1)} \exp \left[-\left(\frac{\sigma}{x}\right)^{\lambda}\right]\left\{1-\exp \left[-\left(\frac{\sigma}{x}\right)^{\lambda}\right]\right\}^{\alpha-1}\left\{-\log \left[1-\exp \left(-\left(\frac{\sigma}{x}\right)^{\lambda}\right)\right]^{\alpha}\right\}^{a-1}}{\Gamma(a)-\gamma\left(a,-\alpha \log \left[1-\exp \left(-\left(\frac{\sigma}{x}\right)^{\lambda}\right)\right]\right)},
$$

respectively. A random variable $\mathrm{X}$ with density (2.2) is denoted by $X \sim G E F(a, \alpha, \lambda, \sigma)$. Plots of the density and hazard rate functions for selected parameter values are displayed in Figures 1-4 and $5-8$, respectively.

\section{Properties}

In this section, we develop some structural properties of the GEF distribution.

\subsection{A useful representation}

A useful expansion for equation (2.2) can be derived using the concept of exponentiated distributions. For an arbitrary baseline cdf $G(x)$, a random variable is said to have the exponentiated- $G$ ("exp-G" for short) distribution with power parameter $a>0$, say $Y_{a} \sim \exp -G(a)$, if its pdf and cdf are given by

$$
h_{a}(x)=a G^{a-1}(x) g(x)
$$

and

$$
H_{a}(x)=G^{a}(x),
$$

respectively. For $a>1$ and $a<1$ and for larger values of $x$, the multiplicative factor a $G(x)^{a-1}$ is greater and smaller than one, respectively. The reverse assertion is also true for smaller values of 
(a)

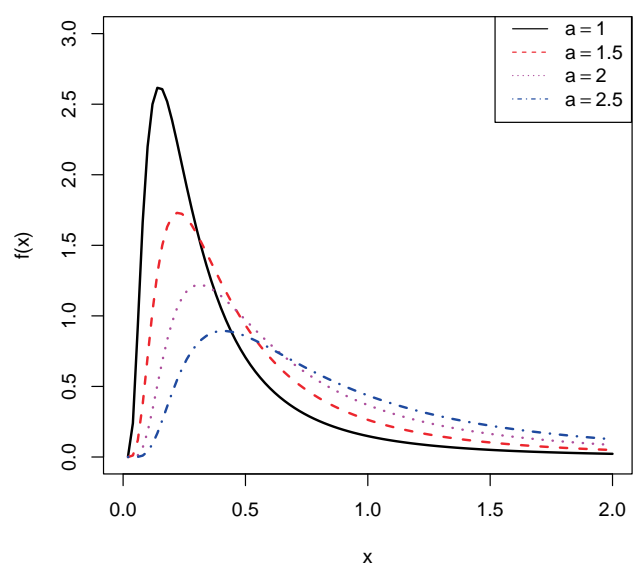

Fig. 1. The GEF density for $\alpha=3, \lambda=0.8$ and $\sigma=$ 0.5 .

(c)

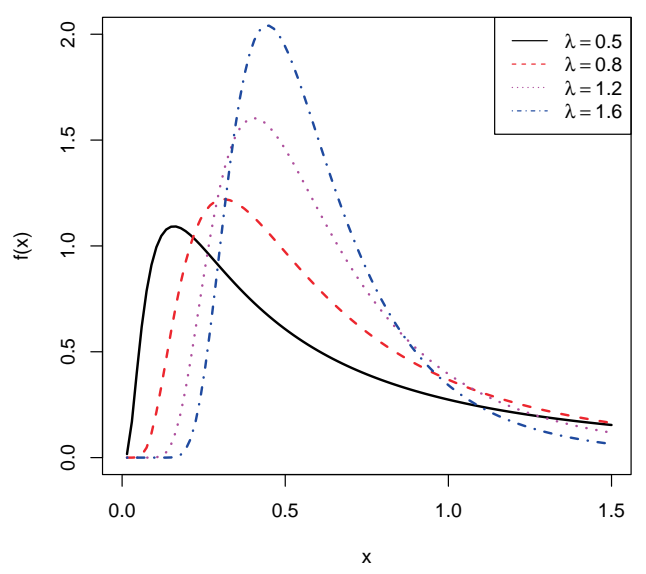

Fig. 3. The GEF density for $a=2, \alpha=3$ and $\sigma=0.5$. (b)

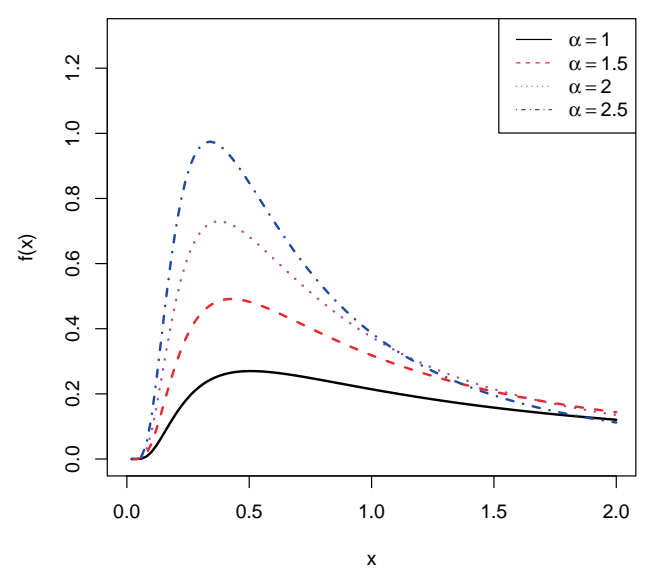

Fig. 2. The GEF density for $a=2, \lambda=0.8$ and $\sigma=$ 0.5 .

(d)

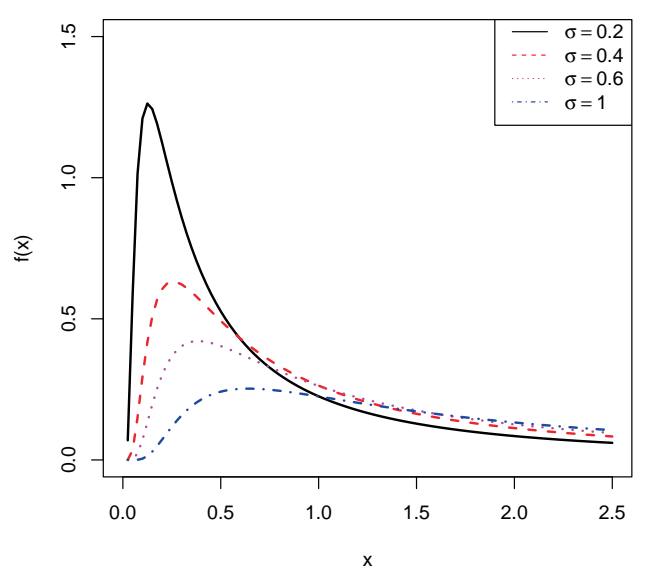

Fig. 4. The GEF density for $a=1.5, \alpha=2$ and $\lambda=$ 0.8 .

$x$. The latter immediately implies that the ordinary moments associated with the density $h_{a}(x)$ are strictly larger (smaller) than those associated with the density $g(x)$, when $a>1(a<1)$.

The properties of exponentiated distributions have been studied by many authors in recent years. See Nadarajah (2005) for exponentiated Gumbel, Kakde and Shirke (2006) for exponentiated lognormal and Nadarajah and Gupta (2007) for exponentiated gamma distributions.

For a real non-integer $a>0$, we define

$$
b_{k}=\frac{\left(\begin{array}{c}
k+1-a \\
k
\end{array}\right)}{(a+k) \Gamma(a-1)} \sum_{j=0}^{k} \frac{(-1)^{j+k}\left(\begin{array}{c}
k \\
j
\end{array}\right) p_{j, k}}{(a-1-j)}
$$


(a)

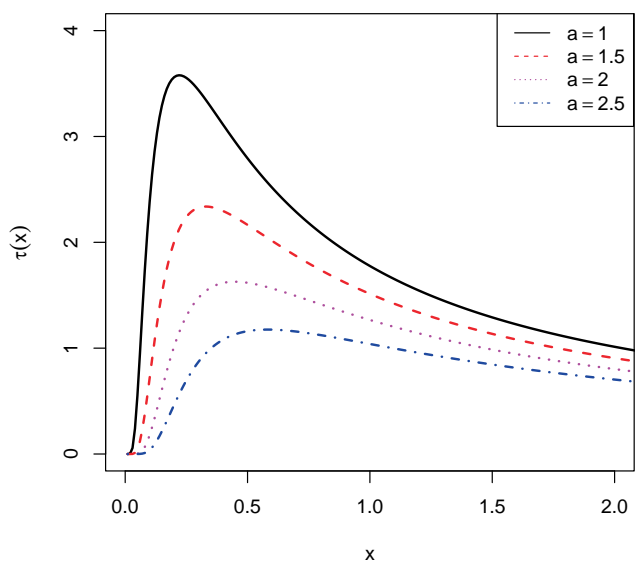

Fig. 5. The GEF hazard rate for $\alpha=3, \lambda=0.8$ and $\sigma=0.5$.

(c)

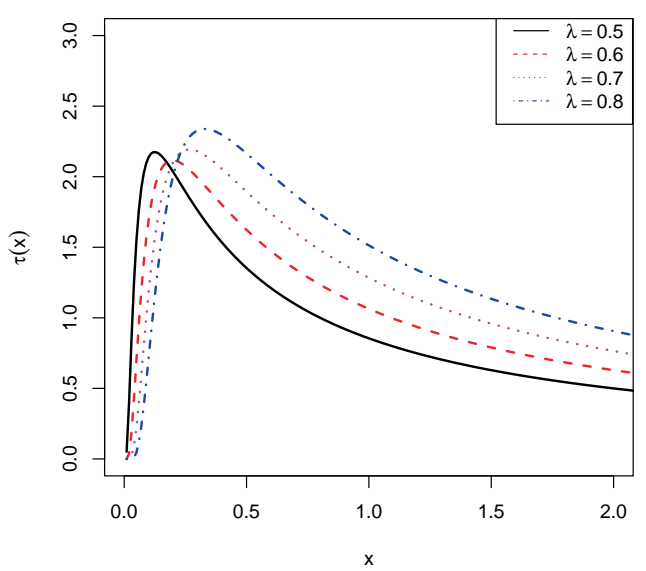

Fig. 7. The GEF hazard rate for $a=1.5, \alpha=3$ and $\sigma=0.5$. (b)

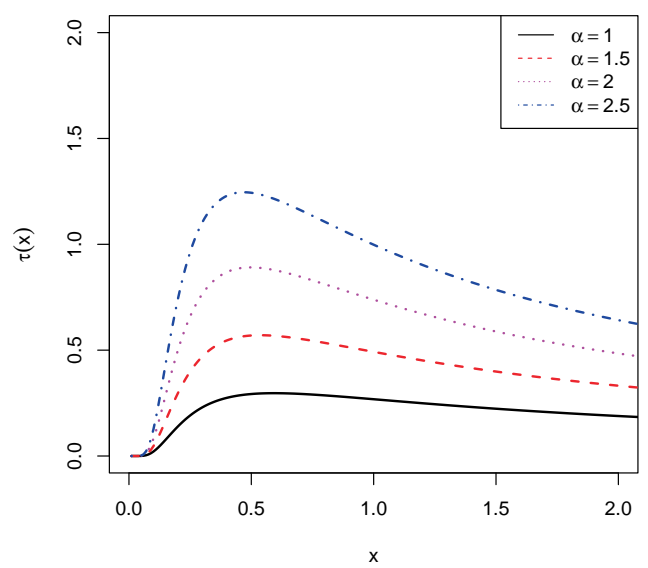

Fig. 6. The GEF hazard rate for $a=2, \lambda=0.8$ and $\sigma=0.5$.

(d)

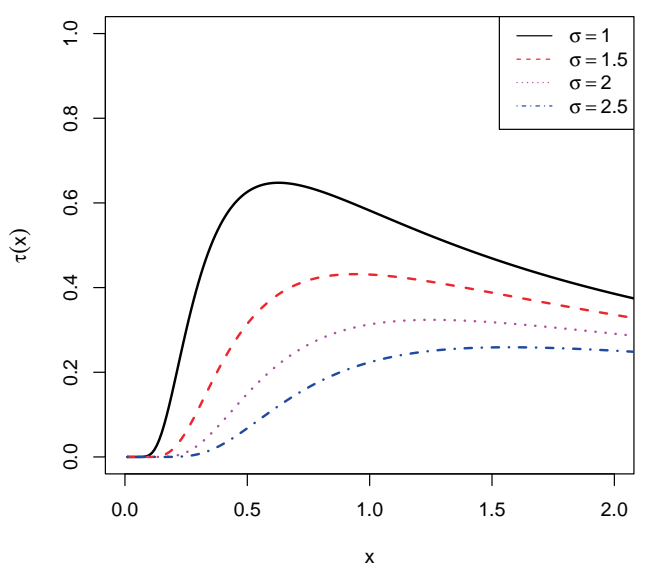

Fig. 8. The GEF hazard rate for $a=1.5, \alpha=2$ and $\lambda=0.8$.

where the constants $p_{j, k}$ can be calculated recursively by

$$
p_{j, k}=k^{-1} \sum_{m=1}^{k} \frac{(-1)^{m}[m(j+1)-k]}{(m+1)} p_{j, k-m}
$$

for $k=1,2, \ldots$ and $p_{j, 0}=1$. Thus, equation (1.5) can be expressed as (Nadarajah et al., 2013)

$$
f(x)=\sum_{k=0}^{\infty} b_{k} h_{a+k}(x)
$$

where $h_{a+k}(x)$ denotes the exp- $G(a+k)$ density function. 
R.V. da Silva et al.

From equation (3.3), we can obtain a simple representation for $f(x)$ as

$$
f(x)=\sum_{i=0}^{\infty} w_{i} g(x ;(i+1) \alpha, \lambda, \sigma)
$$

where

$$
w_{i}=\frac{(-1)^{i}}{(i+1) !} \sum_{k=0}^{\infty} \frac{(a+k) b_{k} \Gamma(a+k)}{\Gamma(a+k-i)}
$$

and $g(x ;(i+1) \alpha, \lambda, \sigma)$ denotes the EF density function given by (4) with parameters $(i+1) \alpha, \lambda$ and $\sigma$. Some details of the proof of (3.4) are given in Appendix A.

\subsection{Quantile function}

From a result presented in Nadarajah et al. (2013), we can generate GEF random variables from the quantile function given by

$$
F^{-1}(u)=\sigma\left\{-\log \left[1-\exp \left(-\alpha^{-1} Q^{-1}(a, 1-u)\right)\right]\right\}^{1 / \lambda},
$$

where $Q^{-1}(a, u)$ is the inverse function of $Q(a, x)=1-\gamma(a, x) / \Gamma(a, x)$, which is available in most statistical packages. Setting $u=1 / 2$ in (3.5), it follows the median $M$ of $X$

$$
M=\sigma\left\{-\log \left[1-\exp \left(-\alpha^{-1} Q^{-1}(a, 1 / 2)\right)\right]\right\}^{1 / \lambda} .
$$

\subsection{Moments}

We now obtain the $n$th moment about zero of $X$, say $E\left(X^{n}\right)$. From equation (3.4), we can write

$$
E\left(X^{n}\right)=\sum_{i=0}^{\infty} w_{i} \int_{0}^{\infty} x^{n} g(x ;(i+1) \alpha, \lambda, \sigma) d x
$$

Setting $y=(\sigma / x)^{\lambda}$ and using equation (4.2) in Nadarajah and Kotz (2003), $E\left(X^{n}\right)$ can be expressed as

$$
\begin{aligned}
E\left(X^{n}\right) & =\frac{\sigma^{n}}{\lambda} \sum_{i=0}^{\infty} w_{i} \int_{0}^{\infty} y^{-\left(\frac{n}{\lambda}+1\right)}\left(1-\mathrm{e}^{-y}\right)^{(i+1) \alpha} d y \\
& =\alpha \sigma^{n} \Gamma\left(1-\frac{n}{\lambda}\right) \sum_{i, r=0}^{\infty} \frac{(-1)^{r+1}(i+1) w_{i}}{(1+r)^{\left(1-\frac{n}{\lambda}\right)}}\left(\begin{array}{c}
(i+1) \alpha-1 \\
r
\end{array}\right) .
\end{aligned}
$$

Next, we derive a simple formula for the $n$th incomplete moment of $X$, say $m_{n}(z)=E\left(X^{n} \mid X<z\right)$. From equation (3.4), we obtain

$$
m_{n}(z)=\sum_{i=0}^{\infty} w_{i} \int_{0}^{z} x^{n} g(x ;(i+1) \alpha, \lambda, \sigma) d x .
$$

Setting $y=(\sigma / x)^{\lambda}$ and after some algebra, the last equation becomes

$$
m_{n}(z)=\frac{\sigma^{n}}{\lambda} \sum_{i=0}^{\infty} w_{i} \int_{0}^{z} y^{-\left(\frac{n}{\lambda}+1\right)}\left(1-\mathrm{e}^{-y}\right)^{(i+1) \alpha} d y
$$


and then

$$
\begin{aligned}
m_{n}(z)= & \alpha \sigma^{n} \sum_{i, r=0}^{\infty} \frac{(-1)^{r+1}(i+1) w_{i}}{(1+r)^{\left(1-\frac{n}{\lambda}\right)}}\left(\begin{array}{c}
(i+1) \alpha-1 \\
r
\end{array}\right) \\
& \times\left\{\left[(1+r) \sigma^{\lambda}\right]^{-\left(1-\frac{n}{\lambda}\right)}-\gamma\left[1-\frac{n}{\lambda},(1+r)\left(\frac{\sigma}{z}\right)^{\lambda}\right]\right\} .
\end{aligned}
$$

\subsection{Mean deviations}

The mean deviations about the mean $\left(\delta_{1}(X)=E\left(\left|X-\mu_{1}^{\prime}\right|\right)\right)$ and about the median $\left(\delta_{2}(X)=\right.$ $E(|X-M|))$ of $X$ can be expressed as

$$
\delta_{1}(X)=2 \mu_{1}^{\prime} F\left(\mu_{1}^{\prime}\right)-2 m_{1}\left(\mu_{1}^{\prime}\right) \quad \text { and } \quad \delta_{2}(X)=\mu_{1}^{\prime}-2 m_{1}(M),
$$

respectively, where $\mu_{1}^{\prime}=E(X)$ comes from (3.6), $M=\operatorname{Median}(X)$ is the median given in Section 3.2, $F\left(\mu_{1}^{\prime}\right)$ is easily calculated from the cdf (2.1) and $m_{1}(z)=\int_{0}^{z} x f(x) d x$ is the first incomplete moment obtained from (3.7) with $n=1$.

Applications of these equations can be given to obtain Bonferroni and Lorenz curves defined for a given probability $\pi$ by

$$
B(\pi)=\frac{m_{1}(q)}{\pi \mu_{1}^{\prime}} \quad \text { and } \quad L(\pi)=\frac{m_{1}(q)}{\mu_{1}^{\prime}},
$$

respectively, where $q=F^{-1}(\pi)$ is the GEF quantile function at $\pi$ determined from (3.5).

\subsection{Rényi entropy}

The entropy of a random variable $X$ with density function $f(x)$ is a measure of variation of the uncertainty. For any real parameter $\omega>0$ and $\omega \neq 1$, the Rényi entropy is given by

$$
\begin{aligned}
I_{R}(\omega)= & \frac{1}{(1-\omega)} \log \int_{0}^{\infty} f^{\omega}(x) d x \\
= & \frac{1}{(1-\omega)} \log \left\{\frac{(a-1) \omega \alpha^{(2 a-1) \omega} \lambda^{\omega-1} \sigma^{\lambda \omega}}{[\Gamma(a)]^{\omega}} \sum_{k, l=0}^{\infty} \sum_{j=0}^{k}(-1)^{k+l+j+1} \frac{\left(\begin{array}{c}
k-(a-1) \omega \\
k
\end{array}\right)\left(\begin{array}{l}
k \\
j
\end{array}\right) p_{j, k}}{(a-1) \omega-j}\right. \\
& \left.\times \frac{[\Gamma[(a-1) \omega+1] \Gamma[\phi]}{l !\left[(k+l+a \omega) \sigma^{\lambda}\right]^{\phi} \Gamma[(a-1) \omega+1-l]}\right\},
\end{aligned}
$$

where $\phi=\frac{\omega(\lambda+1)-1}{\lambda}$. The details of the proof are given in Appendix B.

\subsection{Reliability}

In the context of reliability, the stress-strength model describes the life of a component which has a random strength $X_{1}$ that is subjected to a random stress $X_{2}$. The component fails at the instant that the stress applied to it exceeds the strength, and the component will function satisfactorily whenever $X_{1}>X_{2}$. Hence, $R=P\left(X_{2}<X_{1}\right)=\int_{0}^{\infty} f(x) F(x) d x$ is a measure of component reliability. 
R. V. da Silva et al.

Suppose that $X_{1} \sim$ gamma- $G\left(a_{1}\right)$ and $X_{2} \sim$ gamma- $G\left(a_{2}\right)$ are independent random variables. Let $f_{i}$ and $F_{i}$ be the pdf and cdf of $X_{i}$. Thus, by Nadarajah et al. (2013), we can write

$$
R=\sum_{j, k=0}^{\infty} c_{j k} R_{j k},
$$

where

$$
c_{j k}=\frac{\left(\begin{array}{c}
k+1-a_{1} \\
k
\end{array}\right)}{\left(a_{1}+k\right) \Gamma\left(a_{1}-1\right)} \frac{\left(\begin{array}{c}
j+1-a_{2} \\
j
\end{array}\right)}{\left(a_{2}+j\right) \Gamma\left(a_{2}-1\right)}\left[\sum_{i=0}^{k} \frac{(-1)^{i+k}\left(\begin{array}{c}
k \\
i
\end{array}\right) p_{i, k}}{\left(a_{1}-1-i\right)}\right]\left[\sum_{i=0}^{j} \frac{(-1)^{i+j}\left(\begin{array}{l}
j \\
i
\end{array}\right) p_{i, j}}{\left(a_{2}-1-i\right)}\right],
$$

and $R_{j k}=\operatorname{Pr}\left(Y_{j}<Y_{k}\right)=\int_{0}^{\infty} H_{a_{2}+j}(x) h_{a_{1}+k}(x) d x$ is the reliability between the independent random variables $Y_{j} \sim \exp -G\left(a_{2}+j\right)$ and $Y_{k} \sim \exp -G\left(a_{1}+k\right)$. Hence, the reliability of gamma- $G$ random variables is a linear combination of those of exp- $G$ random variables. In the special case $a_{1}=a_{2}$, equation (3.9) reduces to $R=1 / 2$.

Finally, if $X_{1} \sim G E F\left(a_{1}, \alpha, \lambda, \sigma\right)$ and $X_{2} \sim G E F\left(a_{2}, \alpha, \lambda, \sigma\right)$, using equation (3.9) and after some algebra, we obtain

$$
R=\alpha \sum_{j, k, l, q=0}^{\infty} \frac{(-1)^{l+q+1}\left(a_{1}+k\right) c_{j k} \Gamma[(l+1) \alpha] \Gamma\left[\left(a_{2}+j\right)\left(a_{1}+k\right)+1\right]}{l !(1+q) ! \Gamma[(l+1) \alpha-q] \Gamma\left[\left(a_{2}+j\right)\left(a_{1}+k\right)+1-l\right]}
$$

The details of the proof are given in Appendix C.

\section{Order statistics}

Order statistics make their appearance in many areas of statistical theory and practice. From Nadarajah et al. (2013), the density function $f_{i: n}(x)$ of the $i$ th order statistic $X_{i: n}$, for $i=1, \ldots, n$, from i.i.d. random variables $X_{1}, \ldots, X_{n}$ having an arbitrary $\mathrm{G}$ distribution, is given by

$$
f_{i: n}(x)=\sum_{j=0}^{n-i} \sum_{r, k=0}^{\infty} m_{j, r, k} h_{a(j+i)+r+k}(x)
$$

where

$$
\begin{gathered}
m_{j, r, k}=\frac{(-1)^{j} n !}{(i-1) !(n-i-j) ! j !} \frac{(a+r) b_{r} q_{j+i-1, k}}{[a(j+i)+r+k]}, \\
q_{j+i-1, k}=\left(k b_{0}\right)^{-1} \sum_{m=1}^{k}[m(j+i)-k] b_{m} q_{j+i-1, k-m},
\end{gathered}
$$

and $q_{j+i-1,0}=b_{0}^{j+i-1}$. 
If $X \sim G E F(a, \alpha, \lambda, \sigma)$, we can write after some algebraic manipulations

$$
f_{i: n}(x)=\sum_{q=0}^{\infty} t_{q} g\left(x ; 1, \lambda, \sigma(1+q)^{1 / \lambda}\right),
$$

where

$$
\begin{gathered}
t_{q}=\alpha \sum_{j=0}^{n-i} \sum_{k, r=0}^{\infty}[a(j+i)+k+r] m_{j, k, r} v_{q, j, k, r}, \\
v_{q, j, k, r}=\sum_{l=0}^{\infty} \frac{(-1)^{l+q} \Gamma(l+a) \Gamma[a(j+i)+k+r]}{l !(q+1) ! \Gamma(l+a-q) \Gamma[a(j+i)+k+r-l]},
\end{gathered}
$$

and $g\left(x ; 1, \lambda, \sigma(1+q)^{1 / \lambda}\right)$ denotes the EF density function with parameters one, $\lambda$ and $\sigma(1+q)^{1 / \lambda}$. So, some mathematical properties of the GEF order statistics such as the ordinary and incomplete moments and generating function can be obtained from those of the EF distribution. For example, $E\left(X_{i: n}^{p}\right)=\sigma^{p} \Gamma\left(1-\frac{p}{\lambda}\right) \sum_{q=0}^{\infty}(1+q)^{p} t_{q}$.

\section{Estimation}

Here, we determine the maximum likelihood estimates (MLEs) of the parameters of the new distribution from complete samples only. Let $x_{1}, \ldots, x_{n}$ be a random sample of size $n$ from the GEF $(a, \alpha, \lambda, \sigma)$ distribution. The log-likelihood function can be expressed as

$$
\begin{aligned}
\ell(a, \sigma, \lambda, \alpha) & =-n \log \Gamma(a)+n \log \left(\alpha \lambda \sigma^{\lambda}\right)-\sigma^{\lambda} \sum_{i=1}^{n}\left(x_{i}^{-\lambda}\right)-(\lambda+1) \sum_{i=1}^{n} \log \left(x_{i}\right) \\
& +(a-1) \sum_{i=1}^{n}\left\{\log \left[-\log \left(1-\mathrm{e}^{-z_{i}^{\lambda}}\right)^{\alpha}\right]\right\}+(\alpha-1) \sum_{i=1}^{n}\left[\log \left(1-\mathrm{e}^{-z_{i}^{\lambda}}\right)\right] .
\end{aligned}
$$

The elements of the score vector are given by

$$
\begin{gathered}
U_{a}=n \psi(a)+\sum_{i=1}^{n} \log \left\{-\log \left[1-\mathrm{e}^{-z_{i}^{\lambda}}\right]^{\alpha}\right\}, \\
U_{\alpha}=\frac{n}{\alpha}+\sum_{i=1}^{n} \log \left(1-\mathrm{e}^{-z_{i}^{\lambda}}\right)+(a-1) \sum_{i=1}^{n} \log \left[\log \left(1-\mathrm{e}^{-z_{i}^{\lambda}}\right)\right], \\
U_{\lambda}=\frac{n[1+\lambda \log (\sigma)]}{\lambda}+\sum_{i=1}^{n} \log \left(x_{i}\right)-\sigma^{\lambda} \log (\sigma) \sum_{i=1}^{n}\left(x_{i}^{-\lambda}\right)-\sigma^{\lambda} \sum_{i=1}^{n}\left[-x_{i}^{-\lambda} \log \left(x_{i}\right)\right] \\
+(a-1) \sum_{i=1}^{n}\left[\frac{\alpha \log \left(z_{i}\right) z_{i}^{\lambda} \mathrm{e}^{-z_{i}^{\lambda}}}{\left(1-\mathrm{e}^{-z_{i}^{\lambda}}\right) \log \left(1-\mathrm{e}^{-z_{i}^{\lambda}}\right)}\right]+(\alpha-1) \sum_{i=1}^{n}\left[\frac{\log \left(z_{i}\right) z_{i}^{\lambda} \mathrm{e}^{-z_{i}^{\lambda}}}{\left(1-\mathrm{e}^{-z_{i}^{\lambda}}\right)}\right], \\
U_{\sigma}=\frac{\lambda\left[n-\sigma^{\lambda} \sum_{i=1}^{n}\left(x_{i}^{-\lambda}\right)\right]}{\sigma}+(a-1) \sum_{i=1}^{n}\left[\frac{\alpha \lambda z_{i}^{\lambda} \mathrm{e}^{-z_{i}^{\lambda}}}{\sigma\left(1-\mathrm{e}^{-z_{i}^{\lambda}}\right) \log \left(1-\mathrm{e}^{-z_{i}^{\lambda}}\right)}\right] \\
+(\alpha-1) \sum_{i=1}^{n}\left[\frac{\lambda z_{i}^{\lambda} \mathrm{e}^{-z_{i}^{\lambda}}}{\sigma\left(1-\mathrm{e}^{-z_{i}^{\lambda}}\right)}\right],
\end{gathered}
$$


where $\psi(\cdot)$ is the digamma function.

The MLE $\widehat{\theta}$ of $\theta$ is obtained by solving the nonlinear likelihood equations $U_{a}(\boldsymbol{\theta})=0, U_{\alpha}(\boldsymbol{\theta})=$ $0, U_{\lambda}(\theta)=0$ and $U_{\sigma}(\theta)=0$. These equations cannot be solved analytically and require statistical software with iterative numerical techniques. For interval estimation and hypothesis tests on the parameters $a, \alpha, \lambda$ and $\sigma$, we calculate the observed information matrix since its expectation requires numerical integration. The $4 \times 4$ observed information matrix $J(\theta)$ is

$$
J(\theta)=-\left(\begin{array}{cccc}
\mathbf{U}_{a a} & \mathbf{U}_{a \alpha} & \mathbf{U}_{a \lambda} & \mathbf{U}_{a \sigma} \\
\cdot & \mathbf{U}_{\alpha \alpha} & \mathbf{U}_{\alpha \lambda} & \mathbf{U}_{\alpha \sigma} \\
\cdot & . & \mathbf{U}_{\lambda \lambda} & \mathbf{U}_{\lambda \sigma} \\
\cdot & . & . & \mathbf{U}_{\sigma \sigma}
\end{array}\right) .
$$

The elements of $J(\theta)$ are given in Appendix D.

\section{Application}

In this section, we illustrate the usefulness of the GEF distribution applied to a real data set. We consider the data consisting of the waiting times between 65 consecutive eruptions of the Kiama Blowhole (Pinho et al., 2012). The Kiama Blowhole is a tourist attraction located nearly $120 \mathrm{~km}$ to the south of Sydney. The swelling of the ocean pushes the water through a hole bellow a cliff. The water then erupts through an exit usually drenching whoever is nearby. The times between eruptions of a 1340 hours period starting from July 12th of 1998 were recorded using a digital watch. These data were reported by professor Jim Irish and can be obtained at http://www.statsci.org/data/oz/kiama.html: 83, 51, 87, 60, 28, 95, 8, 27, 15, 10, 18, 16, 29, 54, $91,8,17,55,10,35,47,77,36,17,21,36,18,40,10,7,34,27,28,56,8,25,68,146,89,18,73$, $69,9,37,10,82,29,8,60,61,61,18,169,25,8,26,11,83,11,42,17,14,9,12$.

We fit the density functions of the Beta Fréchet (BF), Weibull (W) and exponentiated Weibull (EW) distributions given by

$$
\begin{gathered}
f_{B F}(x ; a, b, \lambda, \sigma)=\frac{\lambda \sigma^{\lambda}}{B(a, b)} x^{-(\lambda+1)} \exp \left[-a\left(\frac{\sigma}{x}\right)^{\lambda}\right]\left\{1-\exp \left[-\left(\frac{\sigma}{x}\right)^{\lambda}\right]\right\}^{b-1}, \\
f_{W}(x ; \alpha, \lambda)=\alpha \lambda^{\alpha} x^{\alpha-1} \mathrm{e}^{-(\lambda x)^{\alpha}}, \\
f_{E W}(x ; \alpha, \lambda, \gamma)=\alpha \gamma \lambda^{\alpha} x^{\gamma-1} \mathrm{e}^{-(\lambda x)^{\gamma}}\left[1-\mathrm{e}^{-(\lambda x)^{\gamma}}\right]^{\alpha-1},
\end{gathered}
$$

respectively. Now, we apply the Cramér-von Mises $\left(W^{*}\right)$ and Anderson-Darling $\left(A^{*}\right)$ statistics described in details in Chen and Balakrishnan (1995) to verify which distribution fits better to these data. In general, the smaller the values of the statistics $W^{*}$ and $A^{*}$, the better the fit to the data. Let $H(x ; \boldsymbol{\theta})$ be the cdf, where the form of $H$ is known but $\boldsymbol{\theta}$ (a $k$-dimensional parameter vector, say) is unknown. To obtain the statistics $W^{*}$ and $A^{*}$, one can proceed as follows: (i) Compute $v_{i}=H\left(x_{i} ; \widehat{\boldsymbol{\theta}}\right)$, where the $x_{i}$ 's are in ascending order; (ii) Compute $y_{i}=\Phi^{-1}\left(v_{i}\right)$, where $\Phi(\cdot)$ is the standard normal cdf and $\Phi^{-1}(\cdot)$ its inverse; (iii) Compute $u_{i}=\Phi\left\{\left(y_{i}-\bar{y}\right) / s_{y}\right\}$, where $\bar{y}=n^{-1} \sum_{i=1}^{n} y_{i}$ and $s_{y}^{2}=(n-1)^{-1} \sum_{i=1}^{n}\left(y_{i}-\bar{y}\right)^{2}$; (iv) Calculate $W^{2}=\sum_{i=1}^{n}\left\{u_{i}-(2 i-1) /(2 n)\right\}^{2}+1 /(12 n)$ and $A^{2}=-n-(1 / n) \sum_{i=1}^{n}\left\{(2 i-1) \log \left(u_{i}\right)+(2 n+1-2 i) \log \left(1-u_{i}\right)\right\}$; (v) Modify $W^{2}$ into $W^{*}=$ $W^{2}(1+0.5 / n)$ and $A^{2}$ into $A^{*}=A^{2}\left(1+0.75 / n+2.25 / n^{2}\right)$. Table 1 lists the MLEs, their standard errors in parentheses and the statistics $W^{*}$ and $A^{*}$. These statistics indicate that the EW and 
GEF distributions are the best models to these data. Moreover, the standard errors are much smaller compared with their estimates for the GEF distribution.

\begin{tabular}{ccccccc}
\hline Distribution & \multicolumn{3}{c}{ Estimatives } & \multicolumn{3}{c}{ Statistics } \\
\hline GEF & $\widehat{a}$ & $\widehat{\alpha}$ & $\widehat{\lambda}$ & $\widehat{\sigma}$ & $W^{*}$ & $A^{*}$ \\
& 22.98923 & 1.189729 & 4.720202 & 0.47313 & 0.12268 & 0.88974 \\
& $(5.96644)$ & $(0.24741)$ & $(0.18644)$ & $(0.17987)$ & & \\
\hline \hline BF & $\widehat{a}$ & $\widehat{b}$ & $\widehat{\lambda}$ & $\widehat{\sigma}$ & $W^{*}$ & $A^{*}$ \\
& 22.14244 & 0.08067 & 9.65361 & 5.44079 & 3.53847 & 16.7373 \\
& $(9.98259)$ & $(0.01046)$ & $(0.02706)$ & $(0.02423)$ & & \\
\hline \hline $\mathrm{W}$ & & $\widehat{\alpha}$ & $\widehat{\lambda}$ & & $W^{*}$ & $A^{*}$ \\
& - & 1.27478 & 0.023141 & - & 0.14712 & 1.00813 \\
& & $(0.12030)$ & $(0.00239)$ & & & \\
\hline \hline EW & & $\widehat{\alpha}$ & $\widehat{\lambda}$ & $\widehat{\gamma}$ & $W^{*}$ & $A^{*}$ \\
& - & 32.58218 & 1.70136 & 0.35131 & 0.11476 & 0.840963 \\
& & $(89.0288)$ & $(7.94246)$ & $(0.23896)$ & & \\
\hline
\end{tabular}

More information is provided by a visual comparison of the histogram of the data and the fitted densities. The plots of the fitted GEF, BF, W and EW density functions are displayed in Figure 9. These plots indicate that the new distribution provides a good fit to these data and is a very compettitive model to the classical EW distribution.

\section{Conclusions}

We propose a new lifetime model, called the gamma extended Fréchet (GEF) distribution, that generalizes the extended Fréchet $(\mathrm{EF})$ distribution. We provide a mathematical treatment of this distribution. The GEF density function can be expressed as a mixture of EF densities. We derive explicit expressions for the ordinary and incomplete moments, Rényi entropy, mean deviations and reliability. We also obtain the density function of the order statistics. We discuss maximum likelihood estimation and calculate the information matrix. An application to a real data set indicates that the GEF distribution could give better fits than some other common lifetime models. It is also a very competitive model to the exponentiated Weibull distribution.

\section{Acknowledgments}

We are grateful to the anonymous referees and the associated editor for their helpful comments and suggestions on an earlier version of this manuscript which resulted in this improved version.

\section{Appendix A - Simple expansion for the GEF density}

Thus, equation (1.5) can be expressed as

$$
f(x)=\sum_{k=0}^{\infty} b_{k} h_{a+k}(x)
$$

where $h_{a+k}(x)=(a+k) G^{a+k-1}(x) g(x)$ denotes the exp- $G(a+k)$ pdf. 
R.V. da Silva et al.

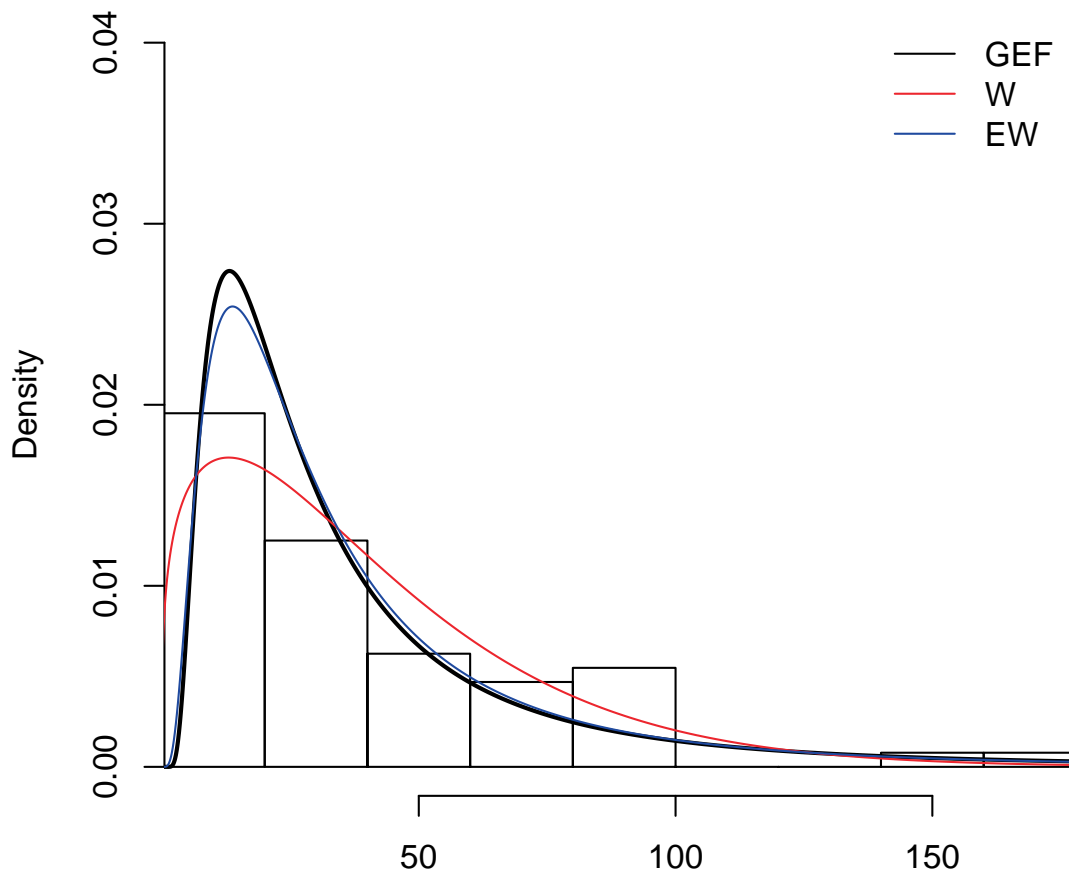

Fig. 9. Fitted densities to the current data.

The density function $h_{a+k}(x)$ can be reduced to

$$
\begin{aligned}
h_{a+k}(x) & =(a+k) \alpha \lambda \sigma^{\lambda} x^{-(\lambda+1)} \mathrm{e}^{-\left(\frac{\sigma}{x}\right)^{\lambda}}\left[1-\mathrm{e}^{-\left(\frac{\sigma}{x}\right)^{\lambda}}\right]^{\alpha-1}\left\{1-\left[1-\mathrm{e}^{-\left(\frac{\sigma}{x}\right)^{\lambda}}\right]^{\alpha}\right\}^{a+k-1} \\
& =(a+k) \alpha \lambda \sigma^{\lambda} x^{-(\lambda+1)} \mathrm{e}^{-\left(\frac{\sigma}{x}\right)^{\lambda}}\left[1-\mathrm{e}^{-\left(\frac{\sigma}{x}\right)^{\lambda}}\right]^{\alpha-1} \sum_{i=0}^{\infty} \frac{(-1)^{i} \Gamma(a+k)}{i ! \Gamma(a+k-i)}\left[1-\mathrm{e}^{-\left(\frac{\sigma}{x}\right)^{\lambda}}\right]^{i \alpha}
\end{aligned}
$$

and then

$$
h_{a+k}(x)=\sum_{i=0}^{\infty} \frac{(-1)^{i} \Gamma(a+k+1)}{(i+1) ! \Gamma(a+k-i)} g(x ;(i+1) \alpha, \lambda, \sigma) .
$$

Thus, $f(x)$ can be written as

$$
\begin{aligned}
f(x) & =\sum_{k=0}^{\infty} b_{k} \sum_{i=0}^{\infty} \frac{(-1)^{i} \Gamma(a+k+1)}{(i+1) ! \Gamma(a+k-i)} g(x ;(i+1) \alpha, \lambda, \sigma) \\
& =\sum_{i=0}^{\infty} w_{i} g(x ;(i+1) \alpha, \lambda, \sigma)
\end{aligned}
$$


where $w_{i}$ is given in Section 3.1 and $g(x ;(i+1) \alpha, \lambda, \sigma)$ denotes the EF density function given by (1.4).

\section{Appendix B - Rényi entropy}

The entropy of a random variable $X$ with density function $f(x)$ is a measure of variation of the uncertainty. For any real parameter $\omega>0$ and $\omega \neq 1$, the Rényi entropy is given by

$$
\begin{aligned}
I_{R}(\omega) & =\frac{1}{(1-\omega)} \log \int_{0}^{\infty} f^{\omega}(x) d x \\
& =\frac{1}{(1-\omega)} \log \int_{0}^{\infty} A(x)\left[1-\mathrm{e}^{-\left(\frac{\sigma}{x}\right)^{\lambda}}\right]^{\omega(\alpha-1)}\left\{-\log \left[1-\mathrm{e}^{-\left(\frac{\sigma}{x}\right)^{\lambda}}\right]^{\alpha}\right\}^{(a-1) \omega} d x
\end{aligned}
$$

where $A(x)=\frac{\alpha^{\omega} \lambda^{\omega} \sigma^{\lambda \omega}}{\Gamma(a)^{\omega}} x^{-\omega(\lambda+1)} \mathrm{e}^{-\omega\left(\frac{\sigma}{x}\right)^{\lambda}}$

Using the expansions

$$
\left[1-\mathrm{e}^{-\left(\frac{\sigma}{x}\right)^{\lambda}}\right]^{(\alpha-1) \omega}=(\alpha-1) \omega \sum_{l=0}^{\infty} \frac{(-1)^{l} \Gamma[(\alpha-1) \omega+1]}{l ! \Gamma[(\alpha-1) \omega-l+1]} \exp \left[-l\left(\frac{\sigma}{x}\right)^{\lambda}\right]
$$

and

$$
\begin{aligned}
\left\{-\log \left[1-\mathrm{e}^{\left.\left.-\left(\frac{\sigma}{x}\right)^{\lambda}\right]\right\}^{(a-1) \omega}}\right.\right. & =(a-1) \omega \sum_{k=0}^{\infty}\left(\begin{array}{c}
k-(a-1) \omega \\
k
\end{array}\right) \sum_{j=0}^{k} \frac{(-1)^{k+j} p_{j, k}\left(\begin{array}{l}
k \\
j
\end{array}\right)}{(a-1) \omega-j} \\
& \times \exp \left\{-[(a-1) \omega+k]\left(\frac{\sigma}{x}\right)^{\lambda}\right\}
\end{aligned}
$$

where $\phi=[\omega(\lambda+1)-1] / \lambda$, we can write $I_{R}(\omega)$ as

$$
\begin{aligned}
I_{R}(\omega)= & \frac{1}{(1-\omega)} \log \left\{\frac{(a-1) \omega \alpha^{(2 a-1) \omega} \lambda^{\omega-1} \sigma^{\lambda \omega}}{[\Gamma(a)]^{\omega}} \sum_{k, l=0}^{\infty} \sum_{j=0}^{k}(-1)^{k+l+j+1} \frac{\left(\begin{array}{c}
k-(a-1) \omega \\
k
\end{array}\right)\left(\begin{array}{l}
k \\
j
\end{array}\right) p_{j, k}}{(a-1) \omega-j}\right. \\
& \left.\times \frac{[\Gamma[(a-1) \omega+1] \Gamma[\phi]}{l !\left[(k+l+a \omega) \sigma^{\lambda}\right]^{\phi} \Gamma[(a-1) \omega-l+1]}\right\} .
\end{aligned}
$$

\section{Appendix C - Reliability}

Nadarajah et al. (2013) demonstrated that

$$
R=\sum_{j, k=0}^{\infty} c_{j k} R_{j k}
$$

where $c_{j k}$ is defined in Section 3.6 and $R_{j k}=\operatorname{Pr}\left(Y_{j}<Y_{k}\right)=\int_{0}^{\infty} H_{a_{2}+j}(x) h_{a_{1}+k}(x) d x$ is the reliability between the independent random variables $Y_{j} \sim \exp -G\left(a_{2}+j\right)$ and $Y_{k} \sim \exp -G\left(a_{1}+k\right)$. Hence, the reliability of gamma- $G$ random variables is a linear combination of those of exp-G random 
R.V. da Silva et al.

variables. We have

$$
H_{a_{2}+j}(x)=G^{a_{2}+j}(x)=\left\{1-\left[1-e^{-\left(\frac{\sigma}{x}\right)^{\lambda}}\right]^{\alpha}\right\}^{a_{2}+j}
$$

and

$$
h_{a_{1}+k}(x)=\left(a_{1}+k\right) \alpha \lambda \sigma^{\lambda} x^{-(\lambda+1)} \mathrm{e}^{-\left(\frac{\sigma}{x}\right)^{\lambda}}\left[1-\mathrm{e}^{-\left(\frac{\sigma}{x}\right)^{\lambda}}\right]^{\alpha-1}\left\{1-\left[1-\mathrm{e}^{-\left(\frac{\sigma}{x}\right)^{\lambda}}\right]^{\alpha}\right\}^{a_{1}+k-1} .
$$

Using the generalized binomial expansion twice, we can write $H_{a_{2}+j}(x) h_{a_{1}+k}(x)$ as

$$
\begin{aligned}
H_{a_{2}+j}(x) h_{a_{1}+k}(x) & =B(x) \mathrm{e}^{-\left(\frac{\sigma}{x}\right)^{\lambda}} \sum_{l=0}^{\infty} \frac{(-1)^{l} \Gamma\left[\left(a_{1}+k\right)+\left(a_{2}+j\right)\right]}{l ! \Gamma\left[\left(a_{1}+k\right)+\left(a_{2}+j\right)-l\right]}\left[1-\mathrm{e}^{-\left(\frac{\sigma}{x}\right)^{\lambda}}\right]^{(l+1) \alpha-1} \\
& =B(x) \sum_{l, q=0}^{\infty} \frac{(-1)^{l+q} \Gamma[(l+1) \alpha] \Gamma\left[\left(a_{1}+k\right)+\left(a_{2}+j\right)\right]}{l ! q ! \Gamma[(l+1) \alpha-q] \Gamma\left[\left(a_{1}+k\right)+\left(a_{2}+j\right)-l\right]} \mathrm{e}^{-(q+1)\left(\frac{\sigma}{x}\right)^{\lambda}}
\end{aligned}
$$

where $B(x)=\left(a_{1}+k\right) \alpha \lambda \sigma^{\lambda} x^{-(\lambda+1)}$. Further, we have

$$
R=\sum_{j, k=0}^{\infty}\left(a_{1}+k\right) c_{j k} \alpha \lambda \sigma^{\lambda} \sum_{l, q=0}^{\infty} \frac{(-1)^{l+q} \Gamma[(l+1) \alpha] \Gamma\left[\left(a_{1}+k\right)+\left(a_{2}+j\right)\right]}{l ! q ! \Gamma[(l+1) \alpha-q] \Gamma\left[\left(a_{1}+k\right)+\left(a_{2}+j\right)-l\right]} I,
$$

where $I=\int_{0}^{\infty} x^{-(\lambda+1)} \mathrm{e}^{-(q+1)\left(\frac{\sigma}{x}\right)^{\lambda}} d x=-\frac{\sigma^{-\lambda}}{(q+1) \lambda}$.

Hence,

$$
R=\alpha \sum_{j, k, l, q=0}^{\infty} \frac{(-1)^{l+q+1}\left(a_{1}+k\right) c_{j k} \Gamma[(l+1) \alpha] \Gamma\left[\left(a_{1}+k\right)+\left(a_{2}+j\right)\right]}{l !(q+1) ! \Gamma[(l+1) \alpha-q] \Gamma\left[\left(a_{1}+k\right)+\left(a_{2}+j\right)-l\right]}
$$

\section{Appendix D - Observed information matrix}

The elements of the observed information matrix $(a, \alpha, \lambda, \sigma)$ are

$$
\begin{gathered}
U_{a a}=n \psi^{\prime}(a), \\
U_{a \lambda}=\sum_{i=1}^{n}\left[\frac{\alpha z_{i}^{\lambda} \log \left(z_{i}\right) \mathrm{e}^{-z_{i}^{\lambda}}}{\left(1-\mathrm{e}^{-z_{i}^{\lambda}}\right) \log \left(1-\mathrm{e}^{-z_{i}^{\lambda}}\right)}\right], \\
U_{a \sigma}=\sum_{i=1}^{n}\left[\frac{\alpha \lambda z_{i}^{\lambda} \mathrm{e}^{-z_{i}^{\lambda}}}{\sigma\left(1-\mathrm{e}^{-z_{i}^{\sigma}}\right) \log \left(1-\mathrm{e}^{-z_{i}^{\lambda}}\right)}\right], \\
U_{\alpha \lambda}=(a-1) \sum_{i=1}^{n}\left[\frac{\log \left(z_{i}\right) z_{i}^{\lambda} \mathrm{e}^{-z_{i}^{\lambda}}}{\left(1-\mathrm{e}^{-z_{i}^{\lambda}}\right) \log \left(1-\mathrm{e}^{-z_{i}^{\lambda}}\right)}\right]+\sum_{i=1}^{n}\left[\frac{\log \left(z_{i}\right) z_{i}^{\lambda} \mathrm{e}^{-z_{i}^{\lambda}}}{\left(1-\mathrm{e}^{-z_{i}^{\lambda}}\right)}\right], \\
U_{\alpha \sigma}=(a-1) \sum_{i=1}^{n}\left[\frac{\lambda z_{i}^{\lambda} \mathrm{e}^{-z_{i}^{\lambda}}}{\sigma\left(1-\mathrm{e}^{-z_{i}^{\lambda}}\right) \log \left(1-\mathrm{e}^{-z_{i}^{\lambda}}\right)}\right]+\sum_{i=1}^{n}\left[\frac{\lambda z_{i}^{\lambda} \mathrm{e}^{-z_{i}^{\lambda}}}{\sigma\left(1-\mathrm{e}^{-z_{i}^{\lambda}}\right)}\right],
\end{gathered}
$$




$$
\begin{aligned}
& U_{\lambda \lambda}=\frac{n\left[2 \alpha \sigma^{\lambda} \log (\sigma)+2 \alpha \lambda \log (\sigma)\right]-n \lambda^{-1}\left[\alpha \sigma^{\lambda}+\alpha \lambda \sigma^{\lambda} \log (\sigma)\right]}{\alpha \lambda \sigma^{\lambda}} \\
& -\frac{n \log (\sigma)\left[\alpha \sigma^{\lambda}+\alpha \lambda \sigma^{\lambda} \log (\sigma)\right]}{\alpha \lambda \sigma^{\lambda}}-2 \log (\sigma) \sigma^{\lambda} \sum_{i=1}^{n} x_{i}^{-\lambda}\left[1-\log \left(x_{i}\right)\right]-\sigma^{\lambda} \sum_{i=1}^{n}\left[2 x_{i}^{-\lambda} \log \left(x_{i}\right)\right] \\
& +(a-1) \sum_{i=1}^{n}\left[\frac{2 \alpha z_{i}^{\lambda} \log \left(z_{i}\right) \mathrm{e}^{-z_{i}^{\lambda}}\left(1-z_{i}^{\lambda}\right)}{\left(1-\mathrm{e}^{-z_{i}^{\lambda}}\right) \log \left(1-\mathrm{e}^{-z_{i}^{\lambda}}\right)}-\frac{3 \alpha \log \left(z_{i}\right) z_{i}^{2 \lambda} \mathrm{e}^{-2 z_{i}^{\lambda}}}{\left(1-\mathrm{e}^{-z_{i}^{\lambda}}\right)^{2} \log \left(1-\mathrm{e}^{-z_{i}^{\lambda}}\right)}\right] \\
& +(\alpha-1) \sum_{i=1}^{n}\left[\frac{2 \log \left(z_{i}\right) z_{i}^{\lambda} \mathrm{e}^{-z_{i}^{\lambda}}\left(1-z_{i}^{\lambda}\right)}{1-\mathrm{e}^{-z_{i}^{\lambda}}}-\frac{2 \log \left(z_{i}\right) z_{i}^{2 \lambda} \mathrm{e}^{-2 z_{i}^{\lambda}}}{\left(1-z_{i}^{\lambda}\right)^{2}}\right] \text {, } \\
& U_{\sigma \sigma}=\frac{-n \lambda+\sigma^{\lambda}\left(\lambda^{2}-\lambda\right) \sum_{i=1}^{n} x_{i}^{-\lambda}}{\sigma^{2}}+(a-1) \sum_{i=1}^{n}\left[\frac{\alpha \lambda z_{i}^{\lambda} \mathrm{e}^{-z_{i}^{\lambda}}(\lambda-1)}{\sigma^{2}\left(1-\mathrm{e}^{-z_{i}^{\lambda}}\right) \log \left(1-\mathrm{e}^{-z_{i}^{\lambda}}\right)}\right. \\
& \left.-\frac{\alpha \lambda^{2} z_{i}^{2 \lambda} \mathrm{e}^{-z_{i}^{\lambda}}\left(2+\mathrm{e}^{-z_{i}^{\lambda}}\right)}{2 \sigma^{2}\left(1-\mathrm{e}^{-z_{i}^{\lambda}}\right) \log \left(1-\mathrm{e}^{-z_{i}^{\lambda}}\right)}-\frac{\alpha \lambda^{2} z_{i}^{2 \lambda} e^{-2 z_{i}^{\lambda}}}{\sigma^{2}\left(1-e^{-z_{i}^{\lambda}}\right)^{2} \log \left(1-e^{-z_{i}^{\lambda}}\right)}\right] \\
& +(\alpha-1) \sum_{i=1}^{n}\left[\frac{\lambda z_{i}^{\lambda} \mathrm{e}^{-z_{i}^{\lambda}}\left(\lambda-1-\lambda z_{i}^{\lambda}\right)}{\sigma^{2}\left(1-\mathrm{e}^{-z_{i}^{\lambda}}\right)}-\frac{\lambda^{2} z_{i}^{2 \lambda} \mathrm{e}^{-2 z_{i}^{\lambda}}}{\sigma^{2}\left(1-\mathrm{e}^{-z_{i}^{\lambda}}\right)^{2}}\right] \\
& U_{\lambda \sigma}=\frac{n-\sigma^{\lambda}[1+\log (\sigma)] \sum_{i=1}^{n}\left(x_{i}^{-\lambda}\right)+\lambda \sigma^{\lambda} \sum_{i=1}^{n} x_{i}^{-\lambda} \log \left(x_{i}\right)}{\sigma} \\
& +(a-1) \sum_{i=1}^{n}\left\{\frac{\alpha z_{i}^{\lambda} \mathrm{e}^{-z_{i}^{\lambda}}\left[1+\lambda \log \left(z_{i}\right)-\lambda z_{i}^{\lambda} \log \left(z_{i}\right)\right]}{\sigma\left(1-\mathrm{e}^{-z_{i}^{\lambda}}\right) \log \left(1-\mathrm{e}^{-z_{i}^{\lambda}}\right)}-\frac{3 \alpha \lambda z_{i}^{2 \lambda} \log \left(z_{i}\right) \mathrm{e}^{-2 z_{i}^{\lambda}}}{2 \sigma\left(1-\mathrm{e}^{-z_{i}^{\lambda}}\right)^{2} \log \left(1-\mathrm{e}^{-z_{i}^{\lambda}}\right)}\right\} \\
& +(\alpha-1) \sum_{i=1}^{n}\left\{\frac{z_{i}^{\lambda} e^{-z_{i}^{\lambda}}\left[1+\lambda \log \left(z_{i}\right)-\lambda z_{i}^{\lambda} \log \left(z_{i}\right)\right]}{\sigma\left(1-\mathrm{e}^{-z_{i}^{\lambda}}\right)}-\frac{\lambda z_{i}^{2 \lambda} \log \left(z_{i}\right) \mathrm{e}^{-2 z_{i}^{\lambda}}}{\sigma\left(1-\mathrm{e}^{-z_{i}^{\lambda}}\right)^{2}}\right\} \text {, }
\end{aligned}
$$

where $z_{i}=\sigma / x_{i}$.

\section{References}

[1] Alves, I.F. and Neves, C. (2010). Extreme value distributions. Available online at http://docentes . deio.fc.ul.pt/fragaalves/fraga_alves_lexicon.pdf.

[2] Barreto-Souza, W., Cordeiro, G.M., Simas, A.B. (2010). Some results for beta Fréchet distribution. Comunications in Statistics - Theory and Methods 40, 798-811.

[3] Chen, G. and Balakrishnan, N. (1995). A general purpose approximate goodness-of-fit test. Journal of Quality Technology 27, 154-161.

[4] Fréchet, M., (1927). Sur la loi de probabilité de l'écart maximum. Annales de la Société de Mathématique 18, 93-116.

[5] Gupta, R.D. and Kundu, D. (1999). Generalized exponential distributions. Australian and New Zealand Journal of Statistics, 41, 173-188.

[6] Gupta, R.D. and Kundu, D. (2001). Exponentiated exponential distribution: an alternative to gamma and Weibull distributions. Biometrical Journal, 43, 117-130.

[7] Kakde, C.S. and Shirke, D.T. (2006). On exponentiated lognormal distribution. International Journal of Agricultural and Statistical Sciences, 2, 319-326.

[8] Mudholkar, G.S. and Srivastava, D.K. (1993). Exponentiated Weibull family for analysing bathtub failure-rate data. IEEE Transactions on Reliability, 42, 299-302. 
R.V. da Silva et al.

[9] Nadarajah, S., Kotz, S. (2003). The exponentiated Fréchet distribution. InterSat. Available online at http://interstat.statjournals . net/YEAR/2003/abstracts/0312001.php.

[10] Nadarajah, S. (2005). The exponentiated Gumbel distribution with climate application. Environmetrics, 17, 13-23.

[11] Nadarajah, S. and Gupta, A.K. (2007). The exponentiated gamma distribution with application to drought data. Calcutta Statistical Association Bulletin, 59, 29-54.

[12] Nadarajah, S., Cordeiro, G.M., Ortega, E.M.M. (2013). The gamma-G family of distributions: Mathematical properties and applications. Communications in Statistics. Theory and Methods. Accepted.

[13] Pal, M., Ali, M.M., Woo, J. (2006). Exponentiated Weibull distribution. Statistica, 2, 139-147.

[14] Pinho, L.G., Cordeiro, G.M., Nobre, J. (2012). The Harris Extended Exponential Distribution. Communications in Statistics-Theory and Methods, accepted.

[15] Ristic, M.M. and Balakrishnan, N. (2011). The gamma exponentiated exponential distribution. Journal of Statistical Computation and Simulation, doi: 10.1080/00949655.2011.574633.

[16] Zografos, K. and Balakrishnan, N. (2009). On families of beta- and generalized gamma-generated distributions and associated inference. Statistical Methodology, 6, 344-362. 